\section{Necessary Condition Analysis (NCA) has either low specificity or low sensitivity}

\section{Kimmo Sorjonen \& Bo Melin}

Department of Clinical Neuroscience,

Karolinska Institutet, Stockholm, Sweden

\section{Abstract}

Objective: To assess the ability of Necessary Condition Analysis (NCA) to achieve its originally stated goal to detect if a condition is necessary but not sufficient for an outcome as well as its more recently stated goal to detect non-randomness in the association between two variables. The performance of NCA was compared with the performance of ordinary linear regression analysis.

Results: For its originally stated goal, NCA exhibited low specificity. For its more recently stated goal, NCA displayed low sensitivity. Ordinary linear regression analysis is superior to NCA for detecting non-random associations. There does not seem to exist any compelling reasons to use the significance test in NCA rather than ordinary linear regression analysis.

Key words: Linear regression analysis, Necessary Condition Analysis (NCA), sensitivity, specificity, statistical performance

\section{Introduction}

Necessary Condition Analysis (NCA) was originally developed to help researchers identify conditions, $\mathrm{X}$, that are necessary but not sufficient for some outcome, $\mathrm{Y}$, of interest. A necessity effect, indicating degree of necessity, is estimated by calculating the size of the empty space in the upper left corner when plotting $\mathrm{X}$ and $\mathrm{Y}$ against each other, as percentage of the square given by $\left(X_{\max }-X_{\min }\right) \times$ $\left(Y_{\max }-Y_{\min }\right)$. An empty space in the upper left corner is seen to indicate that a certain, not too low, degree of $\mathrm{X}$ is necessary for a high degree of $\mathrm{Y}$. Effects below 0.1 have been described as small, effects between 0.1 and 0.3 as medium, effects between 0.3 and 0.5 as large, and effects above 0.5 as very large necessity effects [1]. As an example, the red square given by the lowest and highest values on the $\mathrm{X}$ and $\mathrm{Y}$ variables in Figure 1 has a size equal to 6.081 and the size of the empty space above the ceiling-line in the upper-left corner has a size equal to 2.549. Consequently, the necessity effect (CEFDH) equals $2.549 / 6.081=0.419$. In accordance with its originally stated objective, after conducting NCA researchers have claimed, for example, that a certain, not too low, level of intelligence is necessary but not sufficient for a high level of creativity [2-4].

However, after extending the analysis with a significance test employing permutations [5], the developers of NCA have claimed that the test is for detecting if the association between $\mathrm{X}$ and $\mathrm{Y}$ is

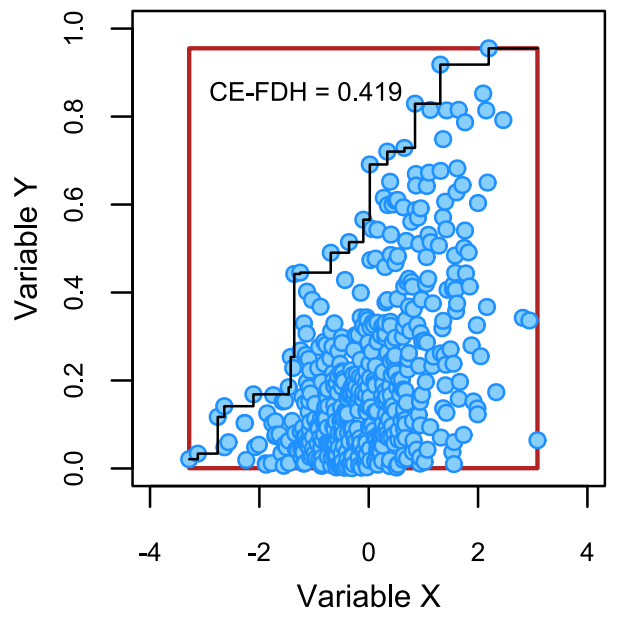

Figure 1. Illustration of the necessity effect (CEFDH) calculated by NCA

characterized by some unspecified type of nonrandomness rather than if $\mathrm{X}$ is necessary but not sufficient for Y [6]. In accordance with this change in objective, the developers have asserted that it is a sign of strength that NCA tends, as demonstrated by Sorjonen and Melin [7, 8], to give significant results when $\mathrm{X}$ is sufficient but not necessary for $\mathrm{Y}$, i.e. the exact opposite of the originally stated objective of NCA [6]. Of course, it might be asked if "A Randomness Test (ART)" or something similar would not be a more appropriate name than "Necessary Condition Analysis (NCA)" given the new objective of the test.

So, the stated objective of NCA has changed from detecting conditions that are necessary but not sufficient for some outcome of interest to detecting if the association between two variables is characterized by some unspecified type of nonrandomness. The objective of the present simulation study was to evaluate how good NCA is at achieving its new goal compared with ordinary linear regression analysis.

Method

Simulations and analyses were conducted with $\mathrm{R}$ 4.1.0 statistical software [9] employing the NCA package [10]. The following steps were utilized: (1) For all sample sizes between 3 and 200, i.e. 198 simulations, virtual participants were allocated a Xscore from a random normal distribution. The participants were then allocated seven $\mathrm{Y}$-scores from different distributions: (a) From a random uniform distribution between 0 and 1 irrespective of their $\mathrm{X}$-score. This tends to give data with no empty corners; (b) From a random beta-distribution with $\alpha$ $=1$ and where $\beta$ decreased from 9 to 1 from the lowest to the highest $\mathrm{X}$-score. This tends to give data with an empty upper-left corner; (c) From a random beta-distribution with $\alpha=1$ and where $\beta$ increased from 1 to 9 from the lowest to the highest $\mathrm{X}$-score. 
This tends to give data with an empty upper-right corner; (d) From a random beta-distribution where $\alpha$ increased from 1 to 9 from the lowest to the highest $\mathrm{X}$-score and with $\beta=1$. This tends to give data with an empty lower-right corner; (e) From a random beta-distribution where $\alpha$ decreased from 9 to 1 from the lowest to the highest $X$-score and with $\beta=1$. This tends to give data with an empty lower-left corner; (f) From a random beta-distribution where $\alpha$ increased from 1 to 9 and $\beta$ decreased from 9 to 1 from the lowest to the highest $\mathrm{X}$-score. This tends to give data with empty upper-left and lower-right corners; (g) From a random beta-distribution where $\alpha$ decreased from 9 to 1 and $\beta$ increased from 1 to 9 from the lowest to the highest $\mathrm{X}$-score. This tends to give data with empty upper-right and lower-left corners. (2) The significance of the association between $\mathrm{X}$ and $\mathrm{Y}$ was calculated with NCA (with 1000 permutations) and with an ordinary linear regression analysis. The association was considered significant if $p<0.05$ and otherwise as nonsignificant. (3) The probability for NCA and linear regression to detect a significant association between $\mathrm{X}$ and $\mathrm{Y}$ was estimated with logistic regression.

\section{Results}

As can be seen in Figure 2, neither NCA nor linear regression tended to indicate a significant association between $\mathrm{X}$ and $\mathrm{Y}$ with higher probability than the nominal 0.05 when there was no association between the two variables (Panel A). NCA tended to be good at detecting associations that were due to an empty upper-left (Panel B) and, to a lower degree, an empty lower-right (Panel D) corner and a combination of these two (Panel F). However, NCA failed completely to detect associations that were due to an empty upper-right (Panel C) and an empty lower-left (Panel E) corner and a combination of these two (Panel G). An ordinary linear regression analysis, on the other hand, was good at detecting all of these associations, unless with a very small sample size.

\section{Discussion}

The present simulations demonstrate that if it is assumed, in accordance with Dul [1], that NCA is for detecting if $\mathrm{X}$ is necessary but not sufficient for $\mathrm{Y}$, the test has low specificity. NCA tends to give significant results even when $\mathrm{X}$ is not necessary for $\mathrm{Y}$, as long as $\mathrm{X}$ is sufficient for $\mathrm{Y}$ (Figure 2, Panel $\mathrm{D})$. On the other hand, if it is assumed, in accordance with Dul et al. [6], that NCA is for detecting if the association between $\mathrm{X}$ and $\mathrm{Y}$ is characterized by some unspecified type of non-randomness, the test has low sensitivity/power. It can be seen in Panels $\mathrm{C}, \mathrm{E}$, and $\mathrm{G}$ in Figure 2 that the test is not able to detect empty spaces in the upper-right and the lowerleft corners of an X-Y-plot. On the other hand, an ordinary least squares (OLS) linear regression analysis has no difficulties detecting these types of deviations from randomness.

An additional advantage of linear regression analysis is that it can include several predictors as well as interaction terms in the same model, something NCA does not seem to be able to handle.

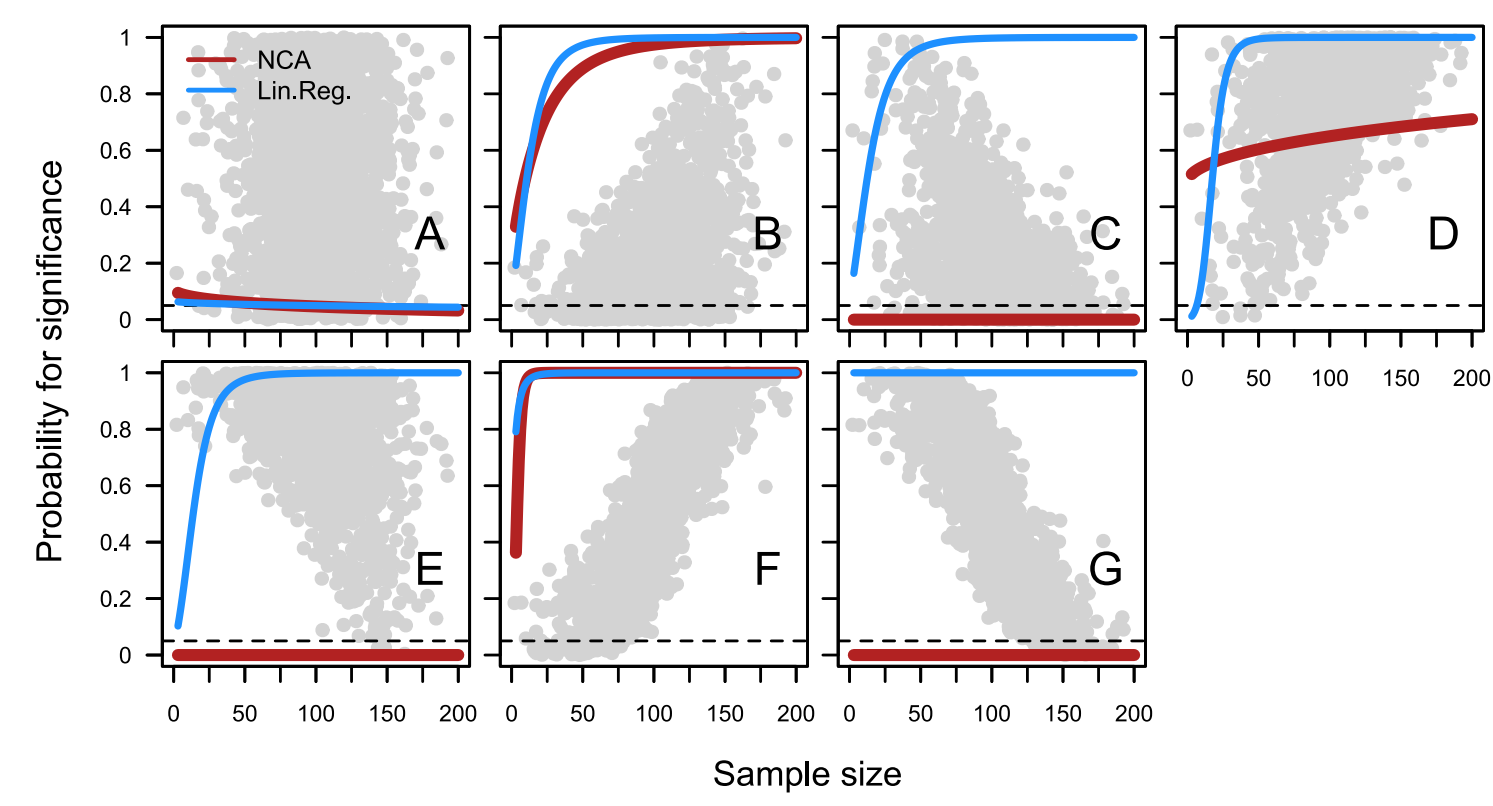

Figure 2. The association between sample size and the ability of NCA and ordinary linear regression analysis to detect non-randomness in the form of an empty space in the upper-left (B), upper-right (C), lower-right (D), lower-left (E), both upper-left and lower-right $(\mathrm{F})$, and both lower-left and upper-right $(\mathrm{G})$ corners, respectively, when plotting two variables against each other. The gray dots in the background illustrate the bivariate distribution that data were drawn from. 
Furthermore, OLS linear regression analysis is computationally much faster. To calculate the significance of the association in Figure 1 with NCA with a recommended 10,000 permutations took approximately 17 seconds on the first author's laptop while an analysis of the same data with linear regression took approximately 0.001 seconds. Taken together, these findings indicate that there does not seem to be any compelling reasons to use the significance test in NCA. It can advantageously be replaced by an ordinary linear regression analysis. However, the size of the necessity effect could possibly still be useful to calculate for descriptive purposes in some situations.

\section{Conclusions}

For its originally stated goal, to identify conditions that are necessary but not sufficient for an outcome, Necessary Condition Analysis (NCA) has low specificity. For its more recently stated goal, to detect non-randomness in the association between two variables, NCA has low sensitivity. Ordinary linear regression analysis is superior to NCA for detecting non-random associations.

\section{Limitations}

Significant results in NCA and regression analyses do not by themselves warrant conclusions about causality. Although the present simulations demonstrated that linear regression is superior to NCA for detecting non-random associations, the data was not ideal for regression analysis. For example, in many cases the analyses resulted in very heteroscedastic residuals. However, this does not affect the conclusion that NCA has very low sensitivity/power to detect some types of associations.

\section{Data availability}

The script, which also generates the data, is available at Open Science Framework: https://osf.io/jqczg/.

\section{References}

1. Dul J. Necessary Condition Analysis (NCA): Logic and methodology of "necessary but not sufficient" causality. Organizational Research Methods. 2016;19:10-52. doi:10.2139/ssrn.2588480.

2. Karwowski M, Dul J, Gralewski J, Jauk E, Jankowska DM, Gajda A, et al. Is creativity without intelligence possible? A Necessary Condition Analysis. Intelligence. 2016;57:105-17. doi:10.1016/j.intell.2016.04.006.

3. Karwowski M, Kaufman JC, Lebuda I, Szumski G, Firkowska-Mankiewicz A. Intelligence in childhood and creative achievements in middle-age: The necessary condition approach. Intelligence. 2017;64:36-44. doi:10.1016/j.intell.2017.07.001.
4. Shi B, Wang L, Yang J, Zhang M, Xu L. Relationship between Divergent Thinking and Intelligence: An Empirical Study of the Threshold Hypothesis with Chinese Children. Front Psychol. 2017;8. doi:10.3389/fpsyg.2017.00254.

5. Dul J, van der Laan E, Kuik R. A statistical significance test for Necessary Condition Analysis. Organizational Research Methods. 2020;23:385-95. doi: $10.1177 / 1094428118795272$

6. Dul J, van der Laan E, Kuik R, Karwowski M. Necessary Condition Analysis: Type I error, power, and over-interpretation of test results. A reply to a comment on NCA. Commentary: Predicting the significance of necessity. Front Psychol. 2019;10:1493. doi:10.3389/fpsyg.2019.01493.

7. Sorjonen K, Melin B. Predicting the significance of necessity. Front Psychol. 2019;10:283. doi:10.3389/fpsyg.2019.00283.

8. Sorjonen K, Melin B. Paradoxical results in and a possible extension of Necessary Condition Analysis (NCA). preprint. PsyArXiv; 2019. doi:10.31234/osf.io/y5xrj.

9. R Core Team. R: A language and environment for statistical computing. R Foundation for Statistical Computing, Vienna, Austria. URL https://www.Rproject.org/. 2021.

10. Dul J. NCA: Necessary Condition Analysis. R package version 3.1.1. https://CRAN.Rproject.org/package=NCA. 2021. 